\title{
Low-level laser and antimicrobial photodynamic therapy on experimental periodontitis in rats submitted to chemotherapy by 5-fluorouracil
}

\author{
Leticia Helena Theodoro $^{1}$ - Mariéllen Longo ${ }^{1}$ - Vivian Cristina Noronha Novaes ${ }^{1}$. \\ Daniela Maria Janjacomo Miessi ${ }^{1} \cdot$ Marcio Luiz Ferro-Alves $^{1} \cdot$ Edilson Ervolino $^{2}$. \\ Juliano Milanezi de Almeida ${ }^{1}$. Valdir Gouveia Garcia ${ }^{1}$
}

Received: 8 February 2017 / Accepted: 24 April 2017 /Published online: 9 May 2017

(C) Springer-Verlag Berlin Heidelberg 2017

\begin{abstract}
Purpose The aim of this study was to evaluate the effects of low-level laser therapy (LLLT) and antimicrobial photodynamic therapy (aPDT) as adjuvant to mechanical treatment of experimental periodontitis (EP) in adult rats submitted to 5-fluorouracil (5-FU) chemotherapy.

Methods EP was induced through ligature around the left mandibular first molar for 7 days. The ligature was removed and the animals separated into groups: $\mathrm{EP}$, no treatment; $5 \mathrm{FU}$, systemic administration of 5-FU ( 80 and $40 \mathrm{mg} / \mathrm{kg}$ ); $5 \mathrm{FU} /$ scaling and root planing (SRP), systemic application of 5FU and SRP; 5FU/SRP/LLLT, systemic application of 5-FU, $\mathrm{SRP}$, and LLLT $\left(660 \mathrm{~nm}, 0.035 \mathrm{~W} ; 29.4 \mathrm{~J} / \mathrm{cm}^{2}\right.$ ); and $5 \mathrm{FU} /$ SRP/aPDT, systemic application of 5-FU, SRP, and aPDT (methylene blue irrigation and LLLT). The animals were euthanized 7, 15, and 30 days after treatments. Histological sections from mandibles were processed for histomorphometric and immunohistochemical analysis (TRAP, RANKL, OPG, TNF- $\alpha$, IL-6, IL-10). The alveolar bone loss (BL) area in the furcation region of the mandibular first molar was analyzed histometrically.

Results There was less bone loss in 5FU/SRP/aPDT compared with $5 \mathrm{FU}$ at 7 days $(p<0.05)$. The immunohistochemical
\end{abstract}

Leticia Helena Theodoro

letheodoro@foa.unesp.br

1 Division of Periodontology, Department of Surgery and Integrated Clinic, School of Dentistry, São Paulo State University (Unesp), Araçatuba, SP, Brazil

2 Division of Histology and Embryology, Department of Basic Science, School of Dentistry, São Paulo State University (Unesp), Araçatuba, SP, Brazil analysis showed no significant difference for TRAP and osteoprotegerin, but lower RANKL immunolabeling was observed in the 5FU/SRP/LLLT and 5FU/SRP/aPDT groups compared with the 5FU group at 15 days. There was lower TNF- $\alpha$ and IL-6 immunolabeling in the 5FU/SRP/LLLT and 5FU/SRP/ aPDT groups and higher IL-10 immunolabeling in 5FU/SRP/ aPDT at 30 days.

Conclusion LLLT and aPDT adjuvant to SRP minimized the effects of 5-FU on periodontal disease. Furthermore, aPDT promoted greater benefits in bone loss control and inflammatory response.

Keywords Periodontitis · Chemotherapy ·

Photochemotherapy $\cdot$ Lasers

\section{Introduction}

Oncologic treatment consists of surgical therapy associated or not with sessions of radiotherapy and/or chemotherapy. Besides the systemic side effects due to immunosuppression, chemotherapy or radiotherapy may also cause undesirable effects in the buccal mucous membrane, such as mucositis, among others [1]. Through the years, chemotherapy has focused on inhibiting proliferation of neoplastic cells, although cancer is considered a disease with a relative deficiency of apoptosis, instead of an excess of cellular proliferation. Therefore, studies have analyzed the effects of the use of chemotherapeutic agents with apoptosis induction potential [2], such as 5-fluorouracil (5-FU) [3]. This drug is indicated for treatment of solid malignant tumors in the gastrointestinal system, central nervous system, breast, and ovary [3], interfering in the metabolism of nucleotides and incorporating into RNA and DNA, leading to cytotoxicity and cellular death [4]. 
Periodontal diseases (PD) are caused by periodontopathogen microorganisms and influenced by local and systemic factors that can alter individual response in the face of microbial aggression [5]. Immune-inflammatory reactions are triggered in periodontal tissues as a way of preventing periodontopathogenic microorganisms or their toxins from spreading or invading the tissues [5]. These reactions constitute the host defense, which in some cases can be harmful, since they can alter or destroy cells in the connective tissue, besides inducing alveolar bone loss [5]. Bacterial infection increases the production of pro-inflammatory cytokines, such as tumoral necrosis factor alfa (TNF- $\alpha$ ) and interleukins, besides inducing the production of other secondary mediators, such as chemokines and prostaglandins [6]. Previous clinical studies pointed out that patients submitted to chemotherapy with 5-FU showed increased gingival inflammation, plaque, and probing depth rate [7-10].

PD treatments involve mechanical removal of the buccal biofilm or maintenance of therapeutic concentrations of antimicrobials in the buccal cavity; however, both procedures present limitations [11, 12]. A recent study demonstrated that 5-FU aggravated periodontitis severity and compromised the host response to a conventional treatment in rats [13], suggesting the necessity of adjuvant therapies.

Low-level laser therapy (LLLT) has been used for treatment and prevention of mucositis induced by chemotherapy, due to the capacity of reducing the severity of these buccal lesions [14-16]. Some in vivo studies evaluated the effects of LLLT as coadjuvant on conventional treatment of experimental periodontitis (EP) in animals with experimentally induced diabetes [17], using immunosupressant drugs [18], nicotine [19], or estrogen suppression [20]. These studies demonstrated some advantages of LLLT, due to its benefits during the tissue repair process. A recent study demonstrated that LLLT is an adjuvant therapy that improves periodontal health in rats with EP under 5-FU chemotherapy [21].

The antimicrobial photodynamic therapy (aPDT) for treatment of human infections is based on the concept that a photosensitizer agent can be absorbed by bacteria and activated by a light with specific wavelength in the presence of oxygen, producing chemical reactions that culminate in the formation of singlet oxygen or free radicals that are cytotoxic for microorganisms [11]. Nevertheless, there are no reports in the literature on the use of aPDT for periodontitis treatment during chemotherapy. Taken together, the purpose of the present study was to compare the effects of LLLT and aPDT, as coadjuvant to scaling and root planing (SRP), to control alveolar bone loss, bone metabolism regulation, and immune-inflammatory response to EP in rats submitted to chemotherapy with 5-FU.

\section{Materials and methods}

\section{Animals}

One hundred fifty adult male rats (Rattus norvegicus, albinus, Wistar) weighing between 200 and $300 \mathrm{~g}$ were used in this study. The experimental protocols (2011-01462) were approved by the Animal Experimentation Ethics Committee of the School of Dentistry of Araçatuba, Sao Paulo State University (UNESP).

\section{Experimental periodontitis}

The animals were anesthetized for all procedures by intramuscular injection of ketamine hydrochloride $(70 \mathrm{mg} / \mathrm{kg}$, Vetaset, Fort Dodge, IA, USA) and xylazine hydrochloride $(6 \mathrm{mg} / \mathrm{kg}$, Coopazine, Coopers, Sao Paulo, SP, Brazil). EP was induced using a cotton thread (\#24, Linha Corrente, São Paulo, SP, Brazil) around the left mandibular first molars. The ligature was kept for 7 days in subgingival position by surgical knots [22].

\section{5-FU treatment}

Systemic treatment with 5 -fluorouracil (5-FU; $50 \mathrm{mg} / \mathrm{ml}$; Eurofarma Laboratórios, Sao Paulo, SP, Brazil) was obtained through initial $(80 \mathrm{mg} / \mathrm{kg})$ and final $(40 \mathrm{mg} / \mathrm{kg}, 48 \mathrm{~h}$ later $)$ intraperitoneal injections [23, 24].

\section{Experimental groups}

Seven days after EP induction, the ligature was removed and the animals were randomly separated, following a computerproduced chart in groups: EP $(n=30)$, no treatment; $5 \mathrm{FU}$ $(n=30)$, treated with 5-FU; 5FU/SRP $(n=30)$, treated with 5-FU, with SRP followed by irrigation with $1 \mathrm{ml}$ of saline solution (SS); 5FU/SRP/LLLT $(n=30)$, treated with 5-FU, with SRP followed by irrigation with SS and LLLT; 5FU/ SRP/aPDT $(n=30)$, treated with 5-FU, with SRP, irrigation with $1 \mathrm{ml}$ of methylene blue (MB) and LLLT.

SRP was carried out with mini-five manual currettes (\#1-2 Hu-Friedy Co. Inc., Chicago, IL, USA) with ten traction movements in the distomesial direction in the vestibular and lingual faces of molars with EP. The interproximal and furcation areas [25] were scraped using the same currettes with traction movements in the cervical-occlusal direction.

\section{LLLT and aPDT}

An indium-gallium-aluminum-phosphorous (InGaAlP) laser (THERA LASE®, D.M.C. Equipments Ltd.®, São Carlos, SP, Brazil) with $660 \mathrm{~nm}$ wavelength and $0.0283 \mathrm{~cm}^{2}$ fiber diameter in continuous operating mode was used. Radiation 
of the left mandibular first molars was carried out with the fiber positioned perpendicularly and almost in contact with the gingival tissue, at $0.035 \mathrm{~W}\left(1.23 \mathrm{~W} / \mathrm{cm}^{2}\right)$ of power, for $12 \mathrm{~s}$ in the center of the vestibular face and $12 \mathrm{~s}$ in the center of the lingual face $(0.84 \mathrm{~J})$, totalizing $24 \mathrm{~s}$ with total energy density of $29.4 \mathrm{~J} / \mathrm{cm}^{2}$ [20].

For aPDT, MB was used as a photosensitizer agent at $100 \mu \mathrm{g} / \mathrm{ml}$ concentration. Using an insulin syringe, $1 \mathrm{ml}$ of MB was deposited in the dento-gingival area of the left mandibular first molar [20]. Sixty seconds after deposition of the photosensitizer, tissue radiation was carried out with laser following the same parameters described above.

\section{Experimental periods}

Ten animals of each group were submitted to euthanasia by injection of a lethal dose of $150 \mathrm{mg} / \mathrm{kg}$ thiopental (Cristália, Produtos Químicos Farmacêuticos Ltda., Itapira, SP, Brazil) at 7, 15, and 30 days after EP. The left hemimandibles were dissected and fixed in $4 \%$ formaldehyde in $0.1 \mathrm{M}$ buffered solution, $\mathrm{pH} 7.4$, for $48 \mathrm{~h}$.

\section{Processing for microscopic analysis}

The specimens were submitted to demineralization in $10 \%$ ethylenediamine tetraacetic acid (EDTA) for 60 days. Subsequently, the specimens were processed by paraffin embedding and sectioned in a microtome ( $4 \mu \mathrm{m}$ thickness). For the histological and histometric analyses, histological sections were submitted to hematoxylin-eosin (HE) staining. For the immunohistochemical analysis, the indirect immunoperoxidase technique was developed using antibodies to TRAP, RANKL, OPG, TNF- $\alpha$, IL-6, and IL-10. The immunohistochemical processing followed the protocol described by Garcia et al. [26].

\section{Histological analysis}

The histological analysis was performed by a certified histologist (EE), who was blinded to all experimental groups. The following parameters were evaluated: nature and degree of inflammation; extent of inflammatory process; presence and extent of tissue necrosis; presence, extent, and nature of bone resorption, cementum, and dentin; blood vessel characteristics; structuring pattern of extracellular matrix of periodontal tissues; and cellularity pattern of periodontal tissues [13].

\section{Histometric analysis}

An imaging analysis system was used (Axiovision 4.8.2, Carl Zeiss MicroImaging GmbH, 07740 Jena, Germany) to measure the bone loss (BL) area in square millimeters in the furcation region. The $\mathrm{BL}$ of each specimen was measured three times at different days by the same examiner, who was calibrated and blinded to treatments (MLFA) in order to reduce data variation. The three obtained measurements were statistically evaluated by analysis of variance at $5 \%$ significance level. The means and standard deviations of absolute values were obtained, and data were compared by statistical analysis [21].

\section{Immunohistochemistry analysis}

TRAP immunolabeling was analyzed by counting TRAPpositive cells located in the center of the interradicular septum of the left mandibular first molar of a $1000-\mu \mathrm{m} \times 1000-\mu \mathrm{m}$ area, at $\times 200$ magnification in three equidistant histological sections. The coronal limit of this area was the alveolar bone ridge, stretching apically along $1000 \mu \mathrm{m}$. Values were expressed as the number of multinucleated TRAP-positive osteoclasts [20].

A semiquantitative analysis of the immunolabeling for RANKL and OPG was performed in the furcation area at $\times 400$ magnification. The establishment of immunolabeling scores was based on Garcia et al., [13]. For TNF- $\alpha$, IL-6, and IL-10, semiquantitative analysis was carried out in three slides per animal and the criterion used was that of Garcia et al. [26].

\section{Statistical analysis}

The results of the calculations for sample size $n$ (minimum) $=10$ demonstrated a statistical power of $95 \%$ $(p<0.05)$. The analyses were performed using software (BioEstat-version 5.3, Instituto Mamirauá, Manaus, AM, Brazil) at $5 \%$ significance level. The normality of the histometric (BL) and immunohistochemical (TRAP) data was analyzed using the Shapiro-Wilk test. BL and TRAP intra- and intergroup analyses were carried out by analysis of variance (ANOVA). When ANOVA detected a statistically significant difference, multiple comparisons were performed using Tukey's post hoc test. The non-parametric KruskalWallis test was used to evaluate RANKL, OPG, IL-6, TNF- $\alpha$, and IL10 score data. This test was followed by the non-parametric Student-Newman-Keuls test, when the Kruskal-Wallis test demonstrated significant difference.

\section{Results}

\section{Histological analysis}

An intense inflammatory infiltrate composed of neutrophils was present in the entire connective tissue of the furcation region in the EP group at 7 days. All specimens of this group presented necrotic bone spicules, surrounded by inflammatory cells in the furcation region. The interradicular septum showed 
a quite irregular outline and thin bone trabeculae, where a large number of active osteoclasts were observed on the surface (Fig. 1a, b).

Inflammation was still very intense at 15 and 30 days. However, comparatively, there was a reduction in the volume occupied by the inflammatory infiltrate constituted of neutrophils and lymphocytes (Figs. 2a, b and 3a, b). The connective tissue surrounding the interradicular septum was less inflamed. The interradicular septum showed thin bone trabeculae, with a quite irregular peripheral outline and active osteoclasts.

The connective tissue of the furcation area presented severe destruction of the extracellular matrix and moderate inflammatory infiltrate that reached the bone tissue of the interradicular septum in group 5FU at 7 and 15 days (Figs. 1c, d and 2c, d). At 30 days, most of the specimens presented necrotic bone spicules, surrounded by inflammatory cells. The interradicular septum bone tissue was composed of very thin bone trabeculae, with extensive medullar spaces presenting inflammatory cells. The peripheral outline of the interradicular septum was very irregular, with many resorption gaps and active osteoclasts (Fig. 3c, d).

There was a moderate inflammatory infiltrate occupying the connective tissue of the furcation area and bordering the bone tissue of the interradicular septum in group 5FU/SRP at 7 days. In most of the specimens, severe damage of the extracellular matrix was observed. Many necrotic bone spicules were present. The interradicular septum showed a quite irregular outline, with the presence of active osteoclasts and constituted of very thin bone trabeculae (Fig. 1e, f). The volume occupied by the inflammatory infiltrate was progressively reduced at 15 and 30 days. The connective tissue surrounding the interradicular septum was significantly immature and inflamed, but it showed discreet repair signs, such as increase in the quantity of collagen fibers, fibroblasts, and blood vessels compared with 5FU. The bone trabeculae were still very thin, with a quite irregular surface, but with fewer osteoclasts and many active osteoblasts (Figs. 2e, f and 3e, f).

Connective tissue with a narrow collagen fiber network, moderate quantity of fibroblasts, and moderate inflammatory infiltrate was observed in group 5FU/SRP/LLLT at 7 days. The interradicular septum was constituted of thin bone trabeculae, and the quantity of osteoclasts was moderate (Fig. 1g, h). The histological characteristics at 15 days were similar to those described in the previous period; however, there was a reduction in the volume occupied by the inflammatory infiltrate and the tissues were more organized (Fig. $2 \mathrm{~g}$, h). At 30 days, the connective tissue was composed of a large number of collagen fibers, with few fibroblasts and isolated focuses of inflammatory cells. The interradicular septum bone tissue was composed of thicker bone trabeculae, with few osteoclasts and many osteoblasts in activity (Fig. 3g, h).

The connnective tissue showed a discreet number of inflammatory cells and a moderate number of collagen fibers
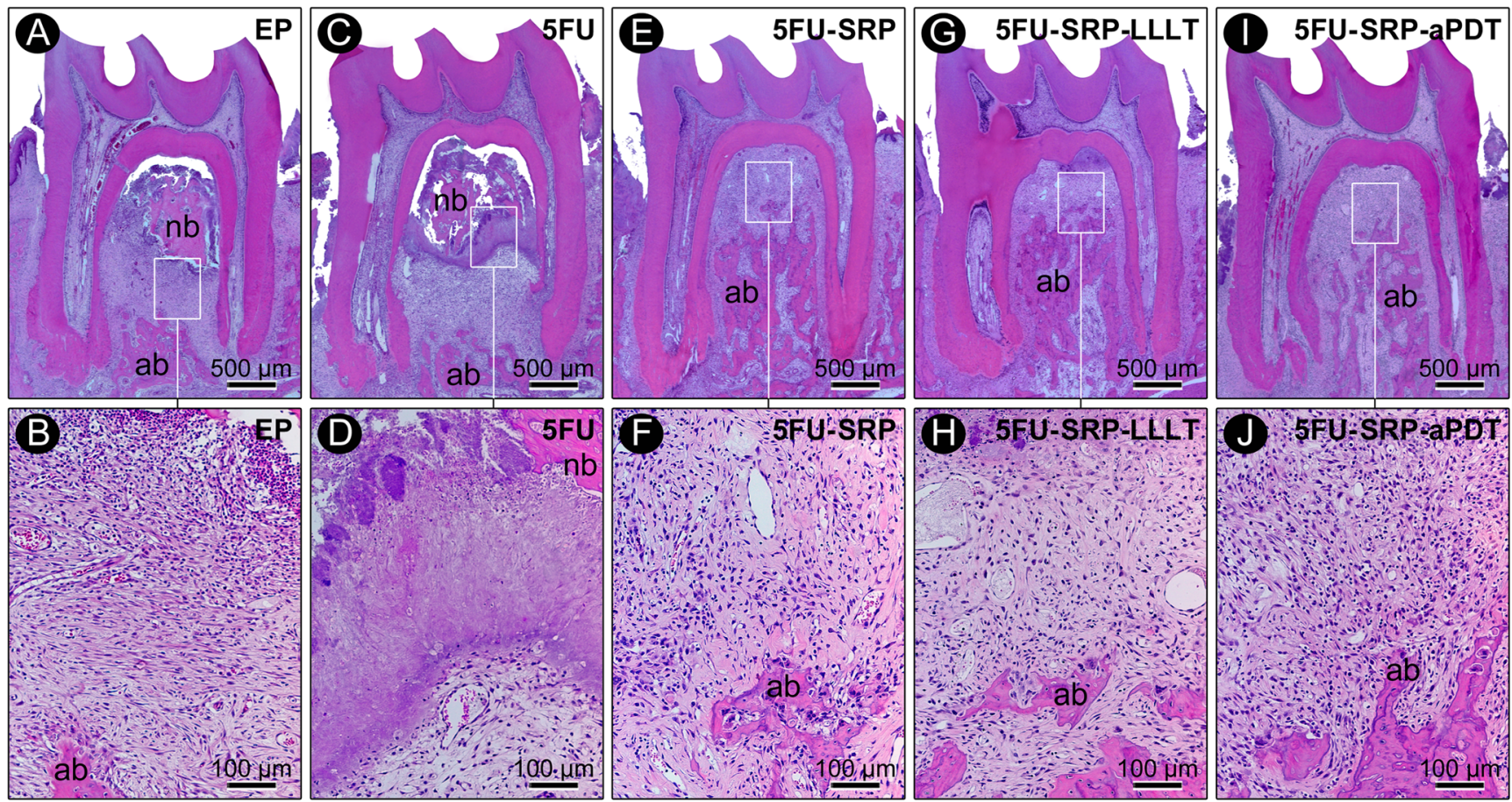

Fig. 1 Photomicrographs of left mandibular first molar with experimental periodontitis showing magnitude of local inflammatory response and level of alveolar bone loss in groups $\mathrm{EP}(\mathbf{a}, \mathbf{b}), 5 \mathrm{FU}(\mathbf{c}$, d), 5FU/SRP $(\mathbf{e}, \mathbf{f}), 5 \mathrm{FU} / \mathrm{SRP} / \mathrm{LLLT}(\mathbf{g}, \mathbf{h})$, and 5FU/SRP/aPDT $(\mathbf{i}, \mathbf{j})$ at

7 days. Abbreviations: $a b$ alveolar bone, $n b$ necrosed bone remains. Original magnification: a, b, e, g, i: $\times 40 ; \mathbf{b}, \mathbf{d}, \mathbf{f}, \mathbf{h}, \mathbf{i}: \times 160$. Scale bars: $\mathbf{a}, \mathbf{c}, \mathbf{e}, \mathbf{g}, \mathbf{i}: 500 \mu \mathrm{m} ; \mathbf{b}, \mathbf{d}, \mathbf{f}, \mathbf{h}, \mathbf{i}: 100 \mu \mathrm{m}$. Staining: hematoxylin and eosin $(\mathrm{H} \& \mathrm{E})$ 

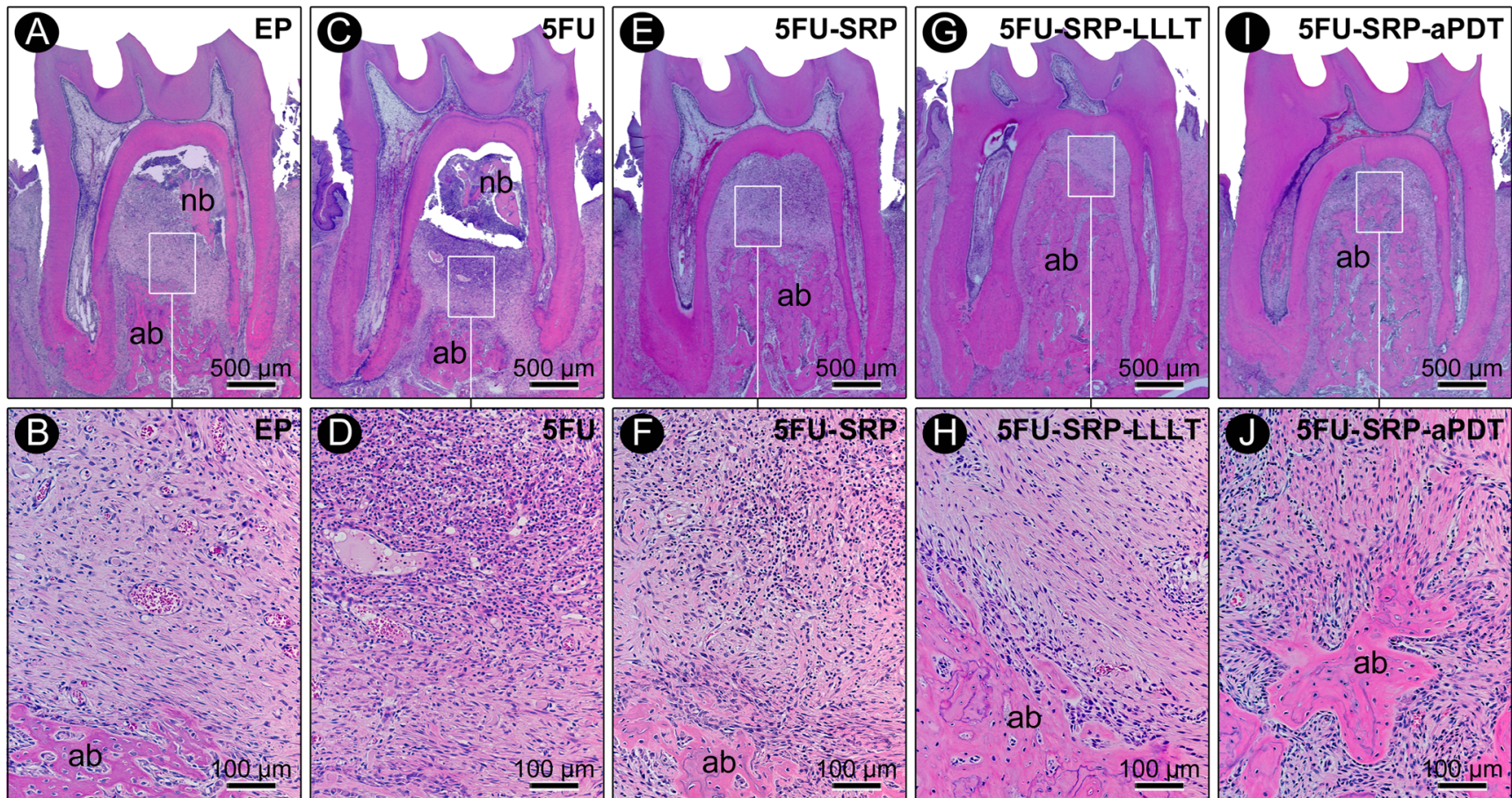

Fig. 2 Photomicrographs of left mandibular first molar with experimental periodontitis showing magnitude of local inflammatory response, level of alveolar bone loss, and alveolar repair process in groups EP (a, b), 5FU (c, d), 5FU/SRP $(\mathbf{e}, \mathbf{f}), 5 \mathrm{FU} / \mathrm{SRP} / \mathrm{LLLT}(\mathbf{g}, \mathbf{h})$, and 5FU/SRP/aPDT (i, j) at 15 days. Abbreviations: $a b$ alveolar bone, $n b$ necrosed bone remains. Original magnification: a, c, e, $\mathbf{g}, \mathbf{i}: \times 40 ; \mathbf{b}, \mathbf{d}, \mathbf{f}, \mathbf{h}$, i: $\times 160$. Scale bars: a, c, e, g, i: $500 \mu \mathrm{m} ; \mathbf{b}, \mathbf{d}, \mathbf{f}, \mathbf{h}, \mathbf{i}: 100 \mu \mathrm{m}$. Staining: hematoxylin and eosin (H\&E) and fibroblasts in group 5FU/SRP/aPDT at 7 days. The interradicular septum had thin bone trabeculae, with few osteoclasts in activity (Fig. 1i, j). At 15 and 30 days, the connective tissue in the furcation region was composed of a
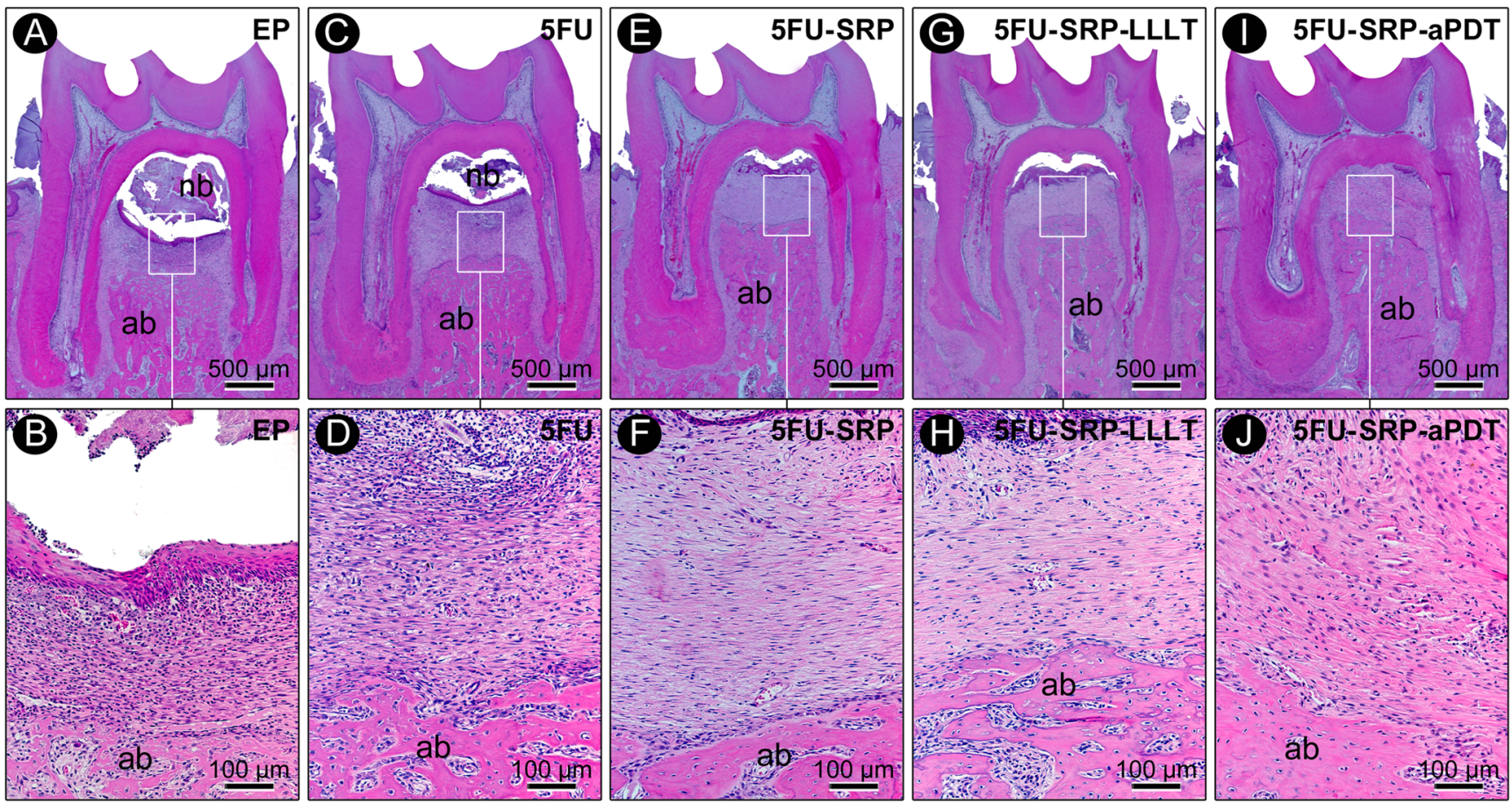

Fig. 3 Photomicrographs of left mandibular first molar with experimental periodontitis showing magnitude of local inflammatory response and level of alveolar bone loss in groups EP (a, b), 5FU (c, d), 5FU/SRP $(\mathbf{e}, \mathbf{f}), 5 \mathrm{FU} / \mathrm{SRP} / \mathrm{LLLT}(\mathbf{g}, \mathbf{h})$, and 5FU/SRP/aPDT $(\mathbf{i}, \mathbf{j})$ at

30 days. Abbreviations: $a b$ alveolar bone, $n b$ necrosed bone remains. Original magnification: a, c, e, $\mathbf{g}, \mathbf{i}: \times 40 ; \mathbf{b}, \mathbf{d}, \mathbf{f}, \mathbf{h}, \mathbf{i}: \times 160$. Scale bars: $\mathbf{a}, \mathbf{c}, \mathbf{e}, \mathbf{g}, \mathbf{i}: 500 \mu \mathrm{m} ; \mathbf{b}, \mathbf{d}, \mathbf{f}, \mathbf{h}, \mathbf{i}: 100 \mu \mathrm{m}$. Staining: hematoxylin and eosin $(\mathrm{H} \& \mathrm{E})$ 
large number of collagen fibers, few fibroblasts, and small isolated focuses of inflammatory cells. The bone trabeculae were thicker and not very irregular, with few osteoclasts and many active osteoblasts (Figs. 2i, j and 3i, j). In some specimens, neoformed bone was observed in the most coronal portion of the interradicular septum.

\section{Histometric analysis}

The results are presented in Table 1 and demonstrated that there was significant smaller alveolar BL in the furcation area in the animals of group 5FU/SRP/aPDT compared with the animals of group $5 \mathrm{FU}$ in the 7 -day evaluation period $(p<0.05)$. In the intragroup evaluation, there were no statistically significant differences in the evaluated periods $(p>0.05)$.

\section{Immunohistochemical analysis}

The immunoreactive cells presented a brownish color enclosed to the cytosolic compartment. For TRAP immunolabeling, a similar pattern prevailed in all groups and periods with no statistically significant difference $(p>0.05)$. TRAP-positive cells were multinucleated and located on the surface of the interradicular septum of the alveolar bone (Fig. 4a, d, g, j, m).

The RANKL and OPG immunolabeling was predominantly expressed in osteoblasts and in some fibroblasts in the bone and connective tissue in the furcation region (Fig. 4b, c, e, f, h, $\mathrm{i}, \mathrm{k}, \mathrm{l}, \mathrm{n}, \mathrm{o}$ ). In the intragroup comparison, there was a significant immunolabeling reduction for RANKL in group $5 \mathrm{FU} /$ SRP/LLLT at $15(p=0.0367)$ and $30(p=0.0191)$ days compared with 7 days. In the intergroup comparison at 15 days, significant immunolabeling reduction was observed for RANKL in groups 5FU/SRP/LLLT $(p=0.0090)$ and 5FU/ $\mathrm{SRP} / \mathrm{aPDT}(p=0.0042)$ compared with group EP and lower immunolabeling in groups 5FU/SRP/LLLT $(p=0.0090)$ and $5 \mathrm{FU} / \mathrm{SRP} / \mathrm{aPDT}(p=0.0042)$ compared with group 5FU. At 30 days, lower RANKL immunolabeling was noticed in

Table 1 Mean \pm SD values of histometric data for BL $\left(\mathrm{mm}^{2}\right)$ in furcation regions of left mandibular first molars according to group and time point

\begin{tabular}{llrl}
\hline Groups & \multicolumn{3}{l}{ Time points } \\
\cline { 2 - 4 } & \multicolumn{1}{l}{ 7 days } & \multicolumn{1}{l}{15 days } & \multicolumn{1}{l}{30 days } \\
\hline EP & $1.16 \pm 0.62$ & $1.6 \pm 0.57$ & $1.13 \pm 0.72$ \\
5FU & $1.86 \pm 0.83$ & $1.85 \pm 0.47$ & $1.50 \pm 0.06$ \\
5FU/SRP & $0.98 \pm 0.49$ & $1.21 \pm 0.54$ & $1.04 \pm 0.42$ \\
5FU/SRP/LLLT & $0.79 \pm 0.19$ & $0.68 \pm 0.35$ & $1.02 \pm 0.68$ \\
5FU/SRP/aPDT & $0.56 \pm 0.28^{\mathrm{a}}$ & $0.67 \pm 0.17$ & $1.01 \pm 0.42$
\end{tabular}

${ }^{\text {a }}$ Statistically significant difference with $5 \mathrm{FU}$ group, in the same time point (ANOVA and Tukey tests, $p<0.05$ )
Fig. 4 Immunolabeling pattern for TRAP, RANKL, and OPG in furcation region of mandibular first molar with experimental periodontitis. Photomicrographs showing immunolabeling for TRAP (a, $\mathbf{d}, \mathbf{g}, \mathbf{j}, \mathbf{m})$, RANKL $(\mathbf{b}, \mathbf{e}, \mathbf{h}, \mathbf{k}, \mathbf{n})$, and OPG $(\mathbf{c}, \mathbf{f}, \mathbf{i}, \mathbf{l}, \mathbf{o})$ at 15 days in groups $\mathrm{EP}(\mathbf{a}, \mathbf{b}, \mathbf{c}), 5 \mathrm{FU}(\mathbf{d}, \mathbf{e}, \mathbf{f}), 5 \mathrm{FU} / \mathrm{SRP}(\mathbf{g}, \mathbf{h}, \mathbf{i}), 5 \mathrm{FU} / \mathrm{SRP} / \operatorname{LLLT}(\mathbf{j}, \mathbf{k}$, l), and 5FU/SRP/aPDT (m, n, o). Abbreviations: $a b$ alveolar bone, arrows immunolabeled cells. Original magnification: $\times 1000$. Scale bars: $25 \mu \mathrm{m}$. Counter-staining: hematoxylin

groups 5FU/SRP $(p=0.0367), 5 \mathrm{FU} / \mathrm{SRP} / \mathrm{LLLT}$ $(p=0.0042)$, and 5FU/SRP/aPDT $(p=0.0042)$ compared with group 5FU. There were no statistically significant intraand intergroup differences for OPG immunolabeling in all groups and evaluated periods $(p=0.0641)$.

TNF- $\alpha$ immunolabeling was predominantly expressed in inflammatory cells and in some fibroblasts in the connective tissue in the furcation region. Regarding TNF- $\alpha$, in the intragroup comparison at 30 days, a significant immunolabeling reduction was observed in groups $\mathrm{EP}(p=0.0123), 5 \mathrm{FU} / \mathrm{SRP}(p=0.0123)$, and 5FU/SRP/LLLT $(p=0.0360)$, compared with the 7-day period. In the intergroup comparison, lower immunolabeling was observed in group 5FU/SRP/aPDT compared with groups $\mathrm{EP}(p=0.0453), 5 \mathrm{FU}(p=0.0453)$, and 5FU/SRP $(p=0.0453)$ at 7 days. Lower immunolabeling was observed in groups $5 \mathrm{FU} /$ SRP/LLLT $(p=0.0051)$ and 5FU/SRP/aPDT $(p=0.0051)$, compared with $5 \mathrm{FU}$ at 15 days. Lower immunolabeling was noticed in groups 5FU/SRP $(p=0.0123), 5 \mathrm{FU} / \mathrm{SRP} / \mathrm{LLLT}$ $(p=0.0019)$, and 5FU/SRP/aPDT $(p=0.0019)$ when compared with group 5FU, as well as in group EP when compared with group 5FU at 30 days ( $p=0.0123$; Fig. $5 \mathrm{a}, \mathrm{d}, \mathrm{g}, \mathrm{j}, \mathrm{m}$ ).

IL-6 immunolabeling was predominantly expressed in inflammatory cells in some fibroblasts in the connective tissue in the furcation region. Regarding IL-6, no statistically significant differences were observed in the intragroup comparison. In the intergroup comparison, lower immunolabeling was noticed in group 5FU/SRP/aPDT when compared with group EP $(p=0.0100), 5 \mathrm{FU}(p=0.0100)$, and 5FU/SRP (0.0100) at 7 days. Lower immunolabeling was also observed in groups 5FU/SRP/LLLT when compared with 5FU $(p=0.0100)$ and 5FU/SRP ( $p=0.0394)$, as well as in group 5FU/SRP/aPDT when compared with groups EP $(p=0.0387), 5 \mathrm{FU}$ $(p=0.0019)$, and 5FU/SRP $(p=0.0098)$ at 15 days. Lower immunolabeling was also noticed in groups $5 \mathrm{FU} / \mathrm{SRP} / \mathrm{LLLT}$ $(p=0.0046)$ and 5FU/SRP/aPDT $(p=0.0046)$ when compared with group $5 \mathrm{FU}$ at 30 days (Fig. $5 \mathrm{~b}, \mathrm{e}, \mathrm{h}, \mathrm{k}, \mathrm{n}$ ).

IL-10 immunolabeling was predominantly expressed in osteoblasts and fibroblasts situated in the bone and connective tissues in the furcation region, respectively. In the IL-10 intragroup analysis in group $5 \mathrm{FU} / \mathrm{SRP} / \mathrm{aPDT}$, higher immunolabeling was observed at 30 days $(p=0.0295)$, compared with the 7-day period. In the intergroup comparison, higher immunolabeling was noticed in group 5FU/SRP/aPDT when compared with groups $\mathrm{EP}(p=0.0295), 5 \mathrm{FU}(p=0.0295)$, and 5FU/SRP at 30 days ( $p=0.0295$; Fig. 5c, f, i, 1, o). 

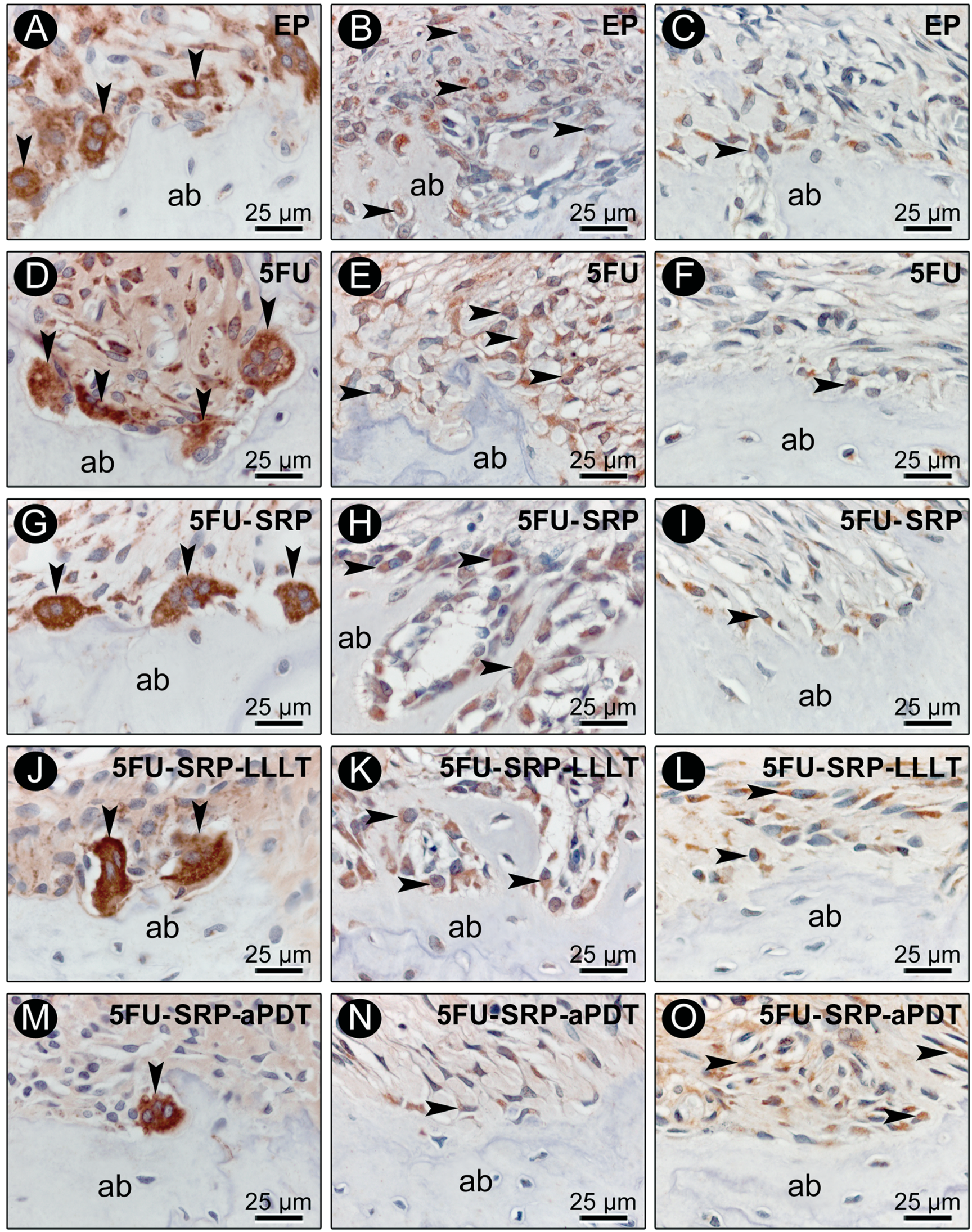

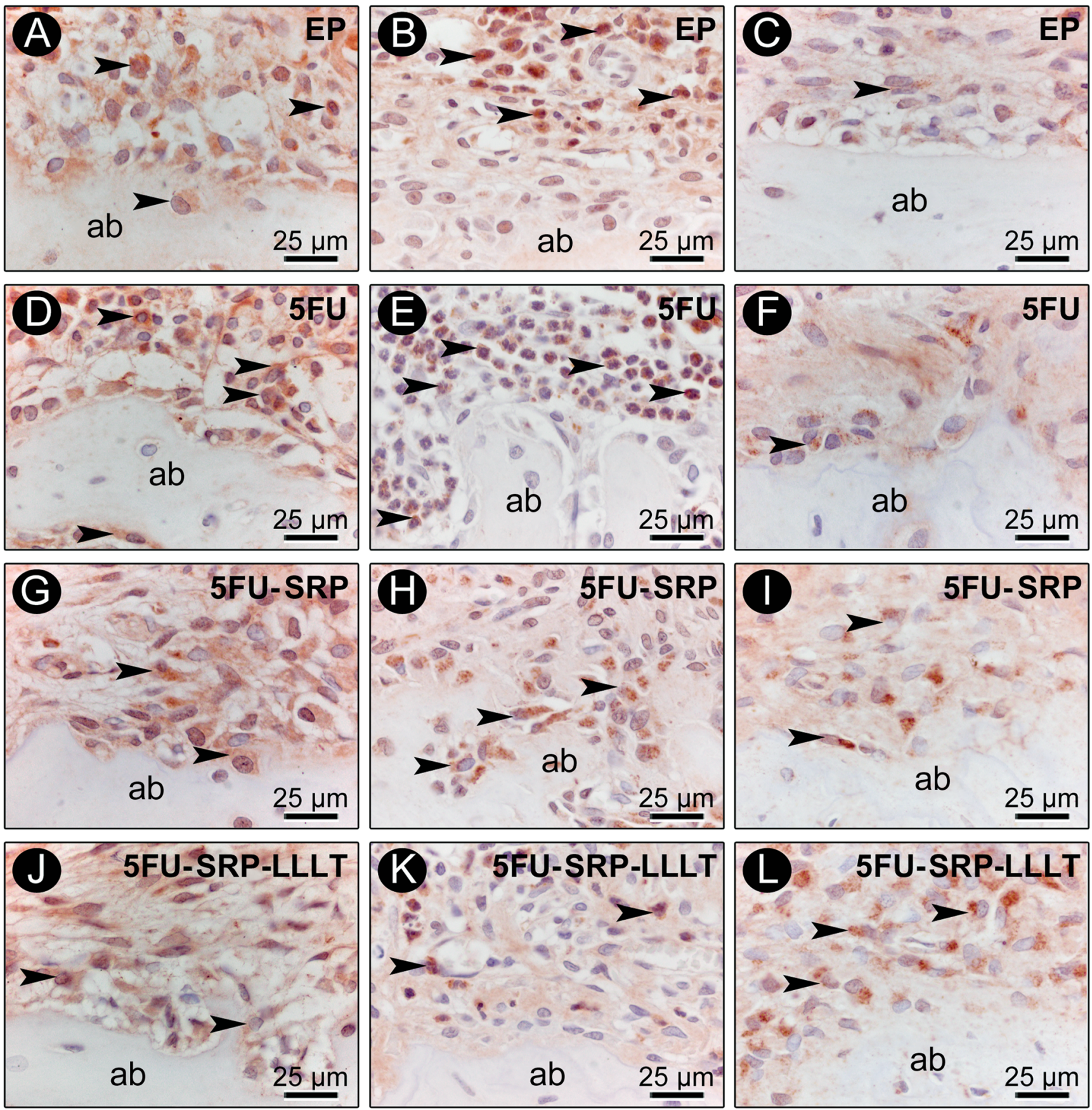

(M) 5FU-SRP-aPDT
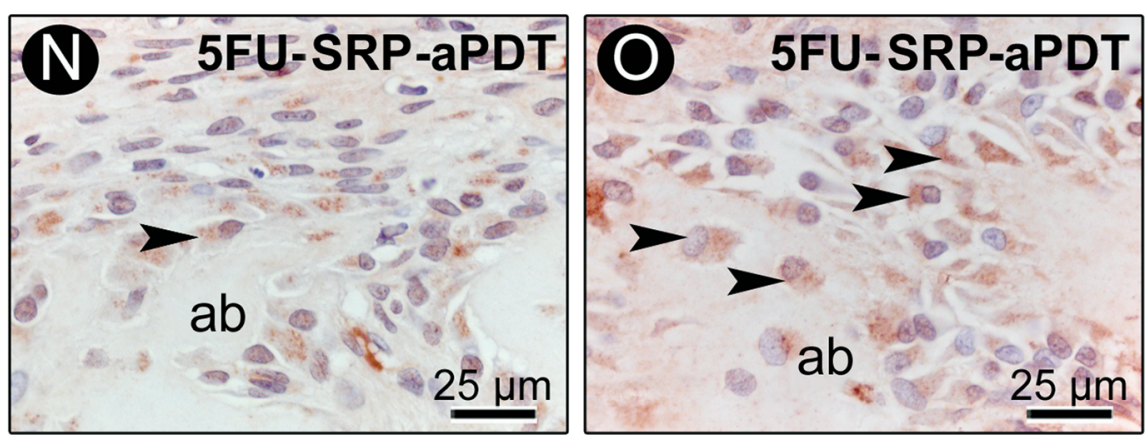
4 Fig. 5 Immunolabeling for TNF- $\alpha$, IL-6, and IL-10 in furcation region of left mandibular first molar with experimental periodontitis. Photomicrographs showing immunolabeling for TNF- $\alpha(\mathbf{a}, \mathbf{d}, \mathbf{g}, \mathbf{j}, \mathbf{m})$ and IL-6 $(\mathbf{b}, \mathbf{e}, \mathbf{h}, \mathbf{k}, \mathbf{n})$ at 7 days and IL-10 (c, f, i, l, o) at 30 days, in groups $\mathrm{EP}(\mathbf{a}, \mathbf{b}, \mathbf{c}), 5 \mathrm{FU}(\mathbf{d}, \mathbf{e}, \mathbf{f}), 5 \mathrm{FU} / \mathrm{SRP}(\mathbf{g}, \mathbf{h}, \mathbf{i}), 5 \mathrm{FU} / \mathrm{SRP} / \mathrm{LLLT}(\mathbf{j}, \mathbf{k}$, l), and 5FU/SRP/aPDT $(\mathbf{m}, \mathbf{n}, \mathbf{o})$. Abbreviations: $a b$ alveolar bone, arrows immunolabeled cells. Original magnification: $\times 1000$. Scale bars: $25 \mu \mathrm{m}$. Counter-staining: hematoxylin

\section{Discussion}

It has been demonstrated that chemotherapy with 5-FU promotes leucopenia in animals [13, 27], increasing the vulnerability of the host to periodontal diseases [6]. The histological analysis demonstrated that EP in animals treated with 5 -FU was more severe, prevailing damage and disorganization of all periodontal tissues, and significant necrotic bone spicules and intense inflammatory infiltrate. However, despite the increase of BL at 7 and 15 days in the animals of group 5FU, there was no statistically significant difference with groups $\mathrm{EP}, 5 \mathrm{FU} /$ SRP, and 5FU/SRP/LLLT. These data demonstrate that 5-FU aggravated the severity of EP, similar to those in studies showing severe alveolar bone damage in rats with EP and submitted to chemotherapy with cyclophosphamide $[28,29]$. These findings are also corroborated by another study where lower bone percentage was observed, with large necrotic bone areas [13] including nearly the totality of the bone in the furcation region in animals submitted to 5-FU chemotherapy. 5-FU induces alterations in the keratinocytes of the oral epithelium [30] via an autophagic degeneration process, affects the local immune response by promoting morphological alterations of the epithelium [31], and significantly reduces the number of osteoblasts of the trabecular surface [32].

Besides in animals treated with aPDT (5FU/SRP/aPDT group), there were significant smaller alveolar $\mathrm{BL}$ in the furcation area compared with animals of group $5 \mathrm{FU}$ in the 7-day evaluation period. On the other hand, the histological analysis of animals in groups 5FU/SRP/LLLT and 5FU/ $\mathrm{SRP} / \mathrm{aPDT}$ revealed connective tissue with a narrow collagen fiber network, a moderate amount of fibroblasts, and moderate inflammatory infiltrate and the interradicular septum was constituted of fine bone trabeculae, with few osteoclasts in the 7-day period. However, most of the animals of the other groups, submitted to chemotherapy (5FU and 5FU/ SRP) and no adjuvant treatments, demonstrated severe damage of the extracellular matrix and presence of many necrotic bone spicules.

These results demonstrated that LLLT and PDT reduced the process of bone tissue damage and local inflammation, favoring the repair process, thus corroborating the findings of other studies in systemically modified animals [17-20]. The effects of LLLT may have biostimulated epithelial migration and organization of the connective tissue $[18,33]$, favoring tissue and bone repair. In this study, lower TNF- $\alpha$ and Il-6 immunolabeling was observed in animals treated with LLLT and aPDT, demonstrating that both evaluated adjuvant therapies were effective in the reduction of these pro-inflammatory biomarkers. The reduction in the levels of these proinflammatory cytokines, in animals treated with LLLT and aPDT, may have interfered in the reduction of the bone damage process that is aggravated by chemotherapy [34].

One possible limitation of this study is lack of a phenotiazinium-photosensitizer-alone treatment group. This fact is justified because several studies of our group did not show significant improvement of periodontitis using phenotiazinium photosensitizers alone in rats $[17,19,22$, 25]. On the other hand, a recent study of our group demonstrated that the combination of multiple LLLT sessions improved inflammation and reduced the destruction of periodontal tissue, worsened by chemotherapy, although it did not reduce periodontopathogenic bacteria of periodontitis areas in rats [21].

Regarding the effects of aPDT, it has been demonstrated that this therapy is able to reduce inflammatory cytokines like IL- $1 \beta$ and TNF- $\alpha[35,36]$. The reduction of proinflammatory biomarkers in this study in animals treated with LLLT and aPDT can be associated with higher reduction of RANKL expression in the 7- and 15-day periods. On the other hand, there were no statistical differences regarding the presence of TRAP- or OPG-positive cells among the evaluated groups. Regarding IL-10 immunolabeling, which is associated with inhibition of bone resorption [37], there was higher immunolabeling in group 5FU/SRP/aPDT compared with groups EP, 5FU, and 5FU/SRP. This finding might be justified by the highest reduction of periodontopathogens in animals treated with aPDT [38], which would reduce the release of pro-inflammatory cytokines, regulating bone metabolism and minimizing the immuno-inflammatory response. Although both evaluated therapies seem effective in the control of EP, further studies are needed to assess the effects of the protocols of these therapies for periodontal treatment in patients under chemotherapy.

\section{Conclusion}

Based on the present results, it is possible to conclude that 5FU aggravated the progression of EP. In addition, LLLT and aPDT were effective adjuvant therapies to SRP, minimizing the side effects caused by 5-FU in periodontal disease. Finally, aPDT promoted significant benefits in the control of alveolar bone loss and immuno-inflammatory response.

Acknowledgements The authors would like to thank the "National Council for Scientific and Technological Development" (CNPq) for the 
research grant and financial support to conduct this study (Process CNPq no. 309447/2010-0).

\section{Compliance with ethical standards}

Ethical approval All procedures performed in the study involving animals were in accordance with the ethical standards of the institution or practice at which the study was conducted.

Conflict of interest The authors declare that they have no conflict of interest.

\section{References}

1. Naidu MU, Ramana GV, Rani PU, Mohan IK, Suman A, Roy P (2004) Chemotherapy-induced and/or radiation therapy-induced oral mucositis - complicating the treatment of cancer. Neoplasia 6(5):423-431

2. Guchelaar HJ, Vermes I, Koopmans RP, Reutelingsperger CP, Haanen C (1998) Apoptosis- and necrosis-inducing potential of cladribine, cytarabine, cisplatin, and 5-fluorouracil in vitro: a quantitative pharmacodynamic model. Cancer Chemother Pharmacol 42(1):77-83

3. Arias JL (2008) Novel strategies to improve the anticancer action of 5-fluorouracil by using drug delivery systems. Molecules 13(10): 2340-2369

4. Noordhuis P, Holwerda U, Van der Wilt CL, Van Groeningen CJ, Smid K, Meijer S, Pinedo HM, Peters GJ (2004) 5-Fluorouracil incorporation into RNA and DNA in relation to thymidylate synthase inhibition of human colorectal cancers. Ann Oncol.15(7): 1025-1032

5. Schenkein HA (2006) Host responses in maintaining periodontal health and determining periodontal diseases. Periodontol 40:77-93

6. Okada H, Murakami S (1998) Cytokine expression in periodontal health and disease. Crit Rev Oral Biol Med 9(3):248-266

7. Jensen SB, Mouridsen HT, Bergmann OJ, Reibel J, Brünner N, Nauntofte B (2008) Oral mucosal lesions, microbial changes, and taste disturbances induced by adjuvant chemotherapy in breast cancer patients. Oral Surg Oral Med Oral Pathol Oral Radiol Endod. 106(2):217-26

8. Mazzeo MA, Linares JA, Campos ML, Busamia BE, Dubersarsky C, Lavarda M, Jarchum G, Finkelberg AB (2009)Oral signs of intravenous chemotherapy with 5-Fluorouracil and Leucovorin calcium in colon cancer treatment. Med Oral Patol Oral Cir Bucal 14(3):E108-13

9. Hong CH, Napeñas JJ, Hodgson BD, Stokman MA, MathersStauffer V, Elting LS, Spijkervet FK, Brennan MT, Dental Disease Section, Oral Care Study Group, Multi-Nacional Association of Supportive Care in Cancer (MASCC)/International Society of Oral Oncology (ISOO) (2010) A systematic review of dental disease in patients undergoing cancer therapy. Support Care Cancer 18(8):1007-1021

10. Djuric M, Cakic S, Hadzi-Mihailovic M, Petrovic D, Jankovic L (2010) Oral status in patients receiving 5-fluorouracil for colorectal cancer. J Buon 15(3):475-479

11. Soukos NS, Goodson JM (2011) Photodynamic therapy in the control of oral biofilms. Periodontol 55(1):143-166

12. Smiley CJ, Tracy SL, Abt E, Michalowicz BS, John MT, Gunsolley J, Cobb CM, Rossmann J, Harrel SK, Forrest JL, Hujoel PP, Noraian KW, Greenwell H, Frantsve-Hawley J, Estrich C, Hanson $N$ (2015) Systematic review and meta-analysis on the nonsurgical treatment of chronic periodontitis by means of scaling and root planing with or without adjuncts. J Am Dent Assoc 146(7):508 524.e5

13. Garcia VG, Novaes VC, De Almeida JM, Longo M, Ervolino E, Bomfim SR, Theodoro LH (2015) Evaluation of the progression and treatment of experimental periodontis in rats subjected to chemotherapy with 5-fluorouracil. Support Care Cancer 23(7):20072017

14. Antunes HS, de Azevedo AM, da Silva Bouzas LF, Adão CA, Pinheiro CT, Mayhe R, Pinheiro LH, Azevedo R, D'aiuto de Matos V, Rodrigues PC, Small IA, Zangaro RA, Ferreira CG (2007) Low-power laser in the prevention of induced oral mucositis in bone marrow transplantation patients: a randomized trial. Blood 109(5):2250-2255

15. Cruz É de P, Campos L, Pereira F da S, Magliano GC, Benites BM, Aranha-Chaves VE, Ballester RY, Simoes A (2015) Clinical, biochemical and histological study of the effect of antimicrobial photodymanic therapy on oral mucositis induced by 5 -fluorouracil in hamster. Photodiagn Photodyn Ther 12(2):298-309

16. França CM, Núñes SC, Prates RA, Noberikawa E, Faria MR, Ribeiro MS (2009) Low-intensity red laser on the prevention and treatment of induced-oral mucositis in hamsters. J Photochem Photobiol B 94(1):25-31

17. de Almeida JM, Theodoro LH, Bosco AF, Nagata MJ, Bonfante S, Garcia VG (2008) Treatment of experimental periodontal disease by photodynamic therapy in rats with diabetes. J Periodontol 79(11):2156-2165

18. Garcia VG, Fernandes LA, de Almeida JM, Bosco AF, Nagata MJ, Martins TM, Okamoto T, Theodoro LH (2010) Comparison between laser therapy and non-surgical therapy for periodontitis in rats treated with dexamethasone. Lasers Med Sci 25(2):197-206

19. Garcia VG, Fernandes LA, Macarini VC, de Almeida JM, Martins TM, Bosco AF, Nagata MJ, Cirelli JA, Theodoro LH (2011) Treatment of experimental periodontal disease with antimicrobial photodynamic therapy in nicotine-modified rats. J Clin Periodontol 38(12):1106-1114

20. Garcia VG, Gualberto Júnior EC, Fernandes LA, Bosco AF, Hitomi Nagata MJ, Casatti CA, Ervolino E, Theodoro LH (2013) Adjunctive antimicrobial photodynamic treatment of experimentally induced periodontitis in rats with ovariectomy. J Periodontol 84(4):556-565

21. Theodoro LH, Longo M, Ervolino E, Duque C, Ferro-Alves ML, Assem NZ, Louzada LM, Garcia VG (2016) Effect of low-level laser therapy as an adjuvant in the treatment of periodontitis induced in rats subjected to 5-fluorouracil chemotherapy. J Periodont Res 51:669-680

22. Garcia VG, Longo M, Gualberto Júnior EC, Bosco AF, Nagata MJ, Ervolino E, Theodoro LH (2014) Effect of the concentration of phenothiazine photosensitizes in antimicrobial photodynamic therapy on bone loss and the immune inflammatory response of induced periodontitis in rats. J Periodontal Res 49(5):584-594

23. Lopes NN, Plapler H, Chavantes MC, Lalla RV, Yosshimura EM, Alves MT (2009) Cyclooxygenase-2 and vascular endothelial growth factor expression in 5-fluorouracil-induced oral mucositis in hamsters: evaluation of two low-intensity laser protocols. Support Care Cancer 17(11):1409-1415

24. Lopes NN, Plapler H, Chavantes MC, Lalla RV, Yosshimura EM, Alves MT (2010) Effects of low-level laser therapy on collagen expression and neutrophil infiltrate in 5-fluorouracil-induced oral mucositis in hamsters. Lasers Surg Med 42(6):546-552

25. Fernandes LA, de Almeida JM, Theodoro LH, Bosco AF, Nagata MJ, Martins TM, Okamoto T, Garcia VG (2009) Treatment of experimental periodontal disease by photodynamic therapy in immunosuppressed rats. J Clin Periodontol 36(3):219-228

26 Garcia VG, Knoll LR, Longo M, Novaes VCN, Assem NZ, Ervolino E, de Toledo BEC, Theodoro LH (2015) Effect of the 
probiotic Saccharomyces cerevisiae on ligature-induced periodontitis in rats. J Periodont Res

27 Medeiros CA, Leitão RF, Macedo RN, Barboza DR, Gomes AS, Nogueira NA, Alencar NM, Ribeiro RA, Brito GA (2011) Effect of atorvastatinon 5-fluorouracil-induced experimental oral mucositis. Cancer Chemother Pharmacol 67(5):1085-1100

28 Samejima Y, Ebisu S, Okada H (1990) Effect of infection with Eikenella corrodens on the progression of ligature-induced periodontitis in rats. J Periodontal Res 25(5):308-315

29 Sallay K, Sanavi F, Ring I, Pham P, Behling UH, Nowotny A (1982) Alveolar bone destruction in the immunosuppressed rat. J Periodontal Res 17(3):263-274

30 Von Bultzingslowen I, Jontell M, Hurst P, Nannmark U, Kardos T (2001) 5-Fluorouracil induces autophagic degeneration in rat oral keratinocytes. Oral Oncol 37(6):537-544

31 Bultzingslowen I, Jontell M (1999) Macrophages, dendritic cells and $\mathrm{T}$ lymphocytes in rat buccal mucosa and dental pulp following 5-fluorouracil treatment. Eur J Oral Sci 107(3):194-201

32 Xian CJ, Cool JC, Pyragius T, Foster BK (2006) Damage and recovery of the bone growth mechanism in young rats following 5fluorouiracil acute chemotherapy. J Cell Biochem 99(6):1688-1704
33 Garcia VG, Macarini VC, de Almeida JM, Bosco AF, Nagata MJ, Okamoto T, Longo M, Theodoro LH (2012) Influence of low-level laser therapy on wound healing in nicotine-treated animals. Lasers Med Sci 27(2):437-443

34 Pesevska S, Nakova M, Gjorgoski I, Angelov N, Ivanovski K, Nares S, Andreana S (2012) Effect of laser on TNF-alpha expression in inflamed human gingival tissue. Lasers Med Sci 27(2):377-381

35 Carvalho AS, Napimoga MH, Coelho-Campos J, Silva-Filho JV, Thedei G (2011) Photodynamic therapy reduces bone resorption and decreased inflammatory response in an experimental rat periodontal disease model. Photomed Laser Surg 29(11):735-740

36 Braham P, Herron C, Street C, Darveau R (2009) Antimicrobial photodynamic therapy may promote periodontal healing through multiple mechanisms. J Periodontol 80(11):1790-1798

37 Cochran DL (2008) Inflammation and bone loss in periodontal disease. J Periodontol 79(8):1569-1576

38 Garcia VG, Longo M, Fernandes LA, Gualberto Júnior EC, Santinoni Cdos S, Bosco AF, Nagata MJ, Theodoro LH (2013) Treatment of experimental periodontitis in rats using repeated adjunctive antimicrobial photodynamic therapy. Lasers Med Sci 28(1): $143-150$ 